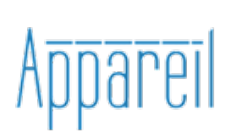

Appareil

11 | 2013

L'espace et l'architecture: état des lieux

\title{
La khôra du Timée : Derrida, lecteur de Platon
}

\section{Marta Hernandez}

\section{OpenEdition}

Journals

Édition électronique

URL : http://journals.openedition.org/appareil/1780

DOI : 10.4000/appareil. 1780

ISSN : 2101-0714

Éditeur

MSH Paris Nord

Référence électronique

Marta Hernandez, "La khôra du Timée : Derrida, lecteur de Platon », Appareil [En ligne], 11 | 2013, mis en ligne le 26 septembre 2013, consulté le 30 juillet 2020. URL : http://journals.openedition.org/ appareil/1780 ; DOI : https://doi.org/10.4000/appareil.1780

Ce document a été généré automatiquement le 30 juillet 2020.

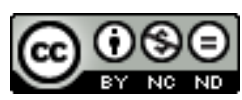

Appareil est mis à disposition selon les termes de la Licence Creative Commons Attribution - Pas d'Utilisation Commerciale - Pas de Modification 4.0 International. 


\title{
La khôra du Timée : Derrida, lecteur de Platon
}

\author{
Marta Hernandez
}

$1 \quad$ Comment parler de l'espace? Et de quel espace? Depuis quelle situation? Depuis quel point de vue qui ne soit pas déjà situé dans l'espace dont il s'agit de parler? Avant de situer l'espace comme l'objet d'une recherche philosophique, d'un discours ou d'une théorie, l'espace nous aurait toujours déjà situés. Pour parler de l'espace sur le mode de l'objectivité ou de la vérité, il faudrait trouver un point de vue qui ne soit pas spatial, un point de vue qui hors de l'espace nous permettrait d'avoir une perspective sur lui, ce qui semble être l'impossible même. C'est de cette impossibilité que, pour Derrida, le discours de Platon sur la khôra aurait témoigné de manière exemplaire.

\begin{abstract}
Le discours sur khôra joue donc pour la philosophie un rôle analogue à celui que joue khôra « elle-même " pour ce dont parle la philosophie, à savoir le cosmos formé ou informé d'après le paradigme. C'est dans ce cosmos qu'on puisera néanmoins les figures propres - mais nécessairement inadéquates - à décrire khôra : réceptacle, porte-empreinte, mère ou nourrice. Ces figures ne sont même pas de vraies figures. De ce qu'elles approchent, la philosophie ne peut parler directement, sur le mode de la vigilance ou de la vérité (vrai ou vraisemblable). Le rêve est entre les deux, ni l'un ni l'autre. La philosophie ne peut parler philosophiquement de ce qui ressemble seulement à sa "mère", sa "nourrice", son "réceptacle» ou son "porteempreinte ». En tant que telle, elle ne parle que du père et du fils, comme si le père l'engendrait à lui tout seul. ${ }^{1}$
\end{abstract}

Le Timée est un récit sur l'origine du monde et, plus précisément, sur la fabrication du monde par le démiurge qui est une sorte d'architecte universel. Le démiurge construit le monde à l'image des paradigmes intelligibles. À la ressemblance des paradigmes, il façonne les êtres sensibles selon un rapport en tout point similaire à celui que le modèle entretient avec la copie et que Platon compare respectivement au père et à l'enfant. De ce dernier, Platon dit qu'il est un mixte (metaxu), une nature intermédiaire entre le père (le modèle) et la mère (la khôra) ${ }^{2}$. Celle-ci représente l'« emplacement ", la "place ", le "réceptacle », le "lieu ». Elle est un troisième genre (triton genus) ${ }^{3}$ qui relève de la nécessité, qui n'est ni sensible ni intelligible et qui précède l'ordre du monde instauré par le démiurgique conformément aux paradigmes intelligibles. Le lieu, 
la khôra, ressortit pour Platon au domaine de l'anánke, qui en grec veut dire aussi bien la " nécessité » que la "contrainte ", d'où l'idée d'une certaine limite, qui rend la khôra inassimilable à l'élément absolument indéterminé et illimité de l'apeiron comme le suggère Léon Robin ${ }^{4}$, aussi bien qu'à l'idée d'un espace abstrait qui rapprocherait la khôra de l'étendue cartésienne comme le veut Heidegger ${ }^{5}$.

Place universelle pour toutes choses, antécédent absolu de tout ordre, Derrida signale que la khôra ne peut être approchée que par un « raisonnement hybride, bâtard (logismô nothô) $»^{6}$ qui ne se laisse pas classer selon l'alternative platonicienne du logos et du mythos.

Par-delà l'opposition arrêtée ou tard venue du logos et du mythos, comment penser la nécessité de ce qui, donnant lieu à cette opposition comme à tant d'autres, semble parfois ne plus se soumettre à la loi de cela même qu'elle situe ? Quoi de ce lieu ? Estil nommable? Et n'aurait-il pas quelque rapport impossible à la possibilité de nommer? Y a-t-il là quelque chose à penser, comme nous le disions si vite, et à penser selon la nécessité ??

Pour parler de cette "réalité", Platon se sert seulement des images et des comparaisons : la khôra est comme la mère, comme le support qui reçoit les empreintes des formes intelligibles, comme le réceptacle ou comme la nourrice des êtres sensibles, etc. Aucune de ces images et comparaisons ne correspond à une définition de la khôra, ni ne saurait être plus adéquate ou plus proche de la vérité qu'une autre ${ }^{8}$. Pourtant, parmi ces images dont Platon se sert, il y en a une, qui donne un indice du fonctionnement de la khôra. En Timée 52e-53a, elle est comparée par Platon aux cribles ou aux tamis qui servent à l'agriculteur à séparer le bon grain du mauvais et à distribuer les «semences ». Les innumérables interprétations du Timée qui ont tenté de situer la khôra dans le système de Platon ont fait abstraction du fait qu'avant qu'elle puisse être située à l'intérieur de ce système comme étant ceci ou cela, elle a un fonctionnement qui consiste justement à situer, à donner une place aux éléments. Le paradoxe réside en ceci qu'avant toute intervention de l'intelligence critique et judicative, le lieu semble procéder à une opération de sélection et de distribution des éléments, un peu comme s'il avait en lui-même, et selon ce que Platon appelle l'ordre de la "nécessité », les critères qui décident de la valeur et de la non-valeur, de la bonne et de la mauvaise semence, du semblable et du dissemblable.

Ce paradoxe de la khôra, cet anachronisme du lieu - qui avant tout acte proprement critique semble procéder à quelque krinein pour séparer le bon grain du mauvais et leur attribuer des places différentes - a obsédé Derrida depuis quasiment le début jusqu'à la fin de son travail. Déjà en 1968, dans "La pharmacie de Platon", suite à quelques remarques sur l'absence au moins apparente de la mère dans le discours de Platon, Derrida annonce le lieu d'une énigme : la khôra du Timée et son rapport à la "cause errante », au « lieu », au " troisième genre », au " porte-empreinte », au " réceptacle », à la «matrice » et à la "mère $»^{9}$. Il faudra attendre vingt ans pour que la première version du livre publié en 1993 sous le nom de Khôra apparaisse dans un recueil d'articles dédiés à Jean-Pierre Vernant ${ }^{10}$. Entre-temps, la khôra aurait fait hommage à sa première manifestation dans $L a$ Dissémination en faisant signe vers son énigme par des incursions régulières, éphémères et disséminées dans de nombreux textes de Derrida. Elle a été aussi l'objet d'au moins deux séminaires, tenus par Derrida en 1972 et en $1985^{11}$. Après 1993, elle accompagne Derrida jusqu'à Voyous, publié quelques mois avant sa mort ${ }^{12}$. 
6 Dans son livre Khôra, Derrida esquisse à peine la figure de la khôra à partir de l'image du crible que nous avons évoqué tout à l'heure. Avant cela, en 1985, la comparaison de la khôra au crible de l'agriculteur était le motif central d'une correspondance entre Derrida et l'architecte Peter Eisenman à propos d'un projet pour le parc de la Villette qui avait précisément pour nom, en allusion à la khôra du Timée, Choral Work. Lors de cette correspondance, Derrida écrit que «le plus énigmatique, ce qui résiste et provoque le plus » dans la lecture qu'il tente du Timée, c'est :

l'allusion à la figure du crible [...], à la chora comme crible [...]. Il y a là, dans le Timée - continue-t-il -, une allusion figurale que je ne sais pas interpréter et qui me paraît pourtant décisive. Elle dit quelque chose comme le mouvement, la secousse [...], le séisme au cours duquel une sélection des forces ou des semences a lieu, un tri, un filtrage là où pourtant le lieu reste impassible, indéterminé, amorphe, etc. Ce passage est, dans le Timée, aussi erratique (me semble-t-il), difficilement intégrable, privé d'origine et de telos manifeste, que cette pièce que nous avons imaginée par notre Choral work. ${ }^{13}$

7 Pour préciser un peu plus le lien entre la khôra avec quelque mécanisme de sélection, dans Khôra, Derrida souligne l'analogie entre le fonctionnement de la khôra et les dispositifs d'assortiment des mariages et de sélection des enfants dont Platon parle largement au Livre $\mathrm{V}$ de la République et qu'il reprend tout au début du Timée. Pour que les enfants naissent avec le meilleur naturel possible, Platon propose aux autorités de « machiner en secret l'assortiment des mariages, à l'aide de certains tirages au sort, de manière que, chacun de son côté, mauvais et bons se trouvent unis à des femmes semblables à eux ${ }^{14}$. Cette analogie qui n'est que formelle, indique pour Derrida le schème d'une structure paradoxale et anachronique. Nous pouvons, au risque de trop simplifier la problématique, formuler naïvement la question dans ces termes : comment se peut-il que le «lieu» dont Platon dit qu'il est un antécédent absolu, dépourvu d'intelligence et étranger à l'ordre de la finalité, semble avoir en lui-même quelque critère pour décider de la distribution des positions, conformément à leur valeur, ne fût-ce à la manière d'un crible qui sépare les semences fortes et fertiles des semences faibles et stériles? Le paradoxe est d'autant plus remarquable que, dans le récit de Timée, l'intervention de la khôra répond à la nécessité méthodologique de reprendre l'explication de l'origine du cosmos depuis le début de l'exposé pour introduire un commencement plus divisé qui prenne en compte le lieu, l'« espèce de la cause errante et la nature de son mouvement propre $\aleph^{15}$. Tout l'effort de Platon aurait consisté à faire passer la sélection qui opère la khôra pour une sélection naturelle. En effet, pour Platon, la khôra relève de la physis comme l'atteste le fait que la khôra n'arrive qu'une seule fois et qu'elle est unique. Pour Platon, il n'y a qu'une seule khôra, d'où la difficulté à concilier le caractère universel et inconditionné de l'espace platonicien avec la signification du mot khôra lui-même qui, en grec, veut dire le territoire, la place occupée, la contrée, la région ou le pays. Sauf à négliger les effets de la langue et le choix des mots, on est conduit à penser qu'entre la khôra comme emplacement universel et la khôra comme territoire, un rapport qui n'est pas exploré par Platon contraint son discours sur le lieu à abandonner les consignes du discours vraisemblable et à indiquer la voie d'un troisième discours où la distinction entre la réalité et la fiction n'est plus assurée :

à peine peut-on y croire - écrit Platon. C'est lui certes que nous apercevons comme en un rêve, quand nous affirmons que tout être est forcément quelque part, en un certain lieu, occupe une certaine place, et que ce qui n'est ni sur la terre, ni quelque part dans le Ciel n'est rien du tout ${ }^{16}$. 
d'Athéna, "L'autochtonie: une topique athénienne", Derrida renvoie à la lecture du Ménexène de Platon pour indiquer le lieu d'une autre analogie entre la khôra du Timée et ce que Loraux appelle la " chôra civique ${ }^{17}$. Dans le Ménexène, le mot khôra désigne la mère-terre-patrie. Elle est aussi bien le lieu de naissance des Athéniens autochtones que le "cimentière " vers lequel ils font retour après la mort pour trouver leur repos dans les lieux familiers. Ce que Derrida remarque davantage, c'est que dans ce dialogue l'hommage à la patrie (à la khôra politique) est inséparable d'un hommage à la richesse naturelle du pays, à la surabondance de la physis et à la situation géographique du territoire. La khôra athénienne se distingue de toutes autres contrées car elle a été - dit Platon - « la première à porter cette nourriture bien humaine qu'est le fruit du blé et de l'orge, dont le genre humain tire son meilleur et son plus bel aliment, de sorte qu'elle a bel et bien elle-même engendré cette espèce vivante ${ }^{18}$.

9 La complicité originaire de la physis avec la culture complique la distinction entre la khôra naturelle et la khôra politique. Le lieu est à la fois une chance de physis et un phénomène de l'esprit humain. Or, ce qui est remarquable dans le Ménexène, c'est que Platon parle du lieu, de la khôra athénienne, comme si elle portait en elle le germe de l'humanité à la différence d'autres territoires qui dépensaient ses fruits à nourrir des bêtes. La générosité nourricière de la physis n'est donc pas sans quelques réserves à l'égard de l'élevage de sa progéniture. Elle ne se donne qu'à la race humaine qu'elle a choisie, parmi toutes les espèces, parce qu'elle était la seule à avoir une valeur en ellemême et à pouvoir multiplier les gains de sa richesse naturelle.

[...] en ce temps lointain où la terre tout entière produisait et faisait croître des êtres de toute espèce, animaux ou plantes, la nôtre s'est montrée stérile et pure de bêtes sauvages, mais s'est choisie parmi les êtres vivants et a engendré l'être humain, qui par son intelligence l'emporte sur les autres tout en étant seul à reconnaitre l'existence d'une justice et des dieux $»^{19}$.

10 La khôra athénienne est le lieu d'une sélection entre l'être humain et l'animalité sauvage. À la manière dont la khôra du Timée procédait à la séparation du bon grain et du mauvais, la khôra athénienne exclut de son territoire la mauvaise semence (l'animal) pour faire place à la bonne (l'humain), comme si elle avait déjà en elle-même les critères de la valeur et de la justice humaine. De là, un anachronisme qui n'est pas accidentel, un anachronisme qui est un fait de structure et qui nous contraint à penser l'espace comme quelque chose qui, en nous précédant, serait déjà toujours fait pour nous, prêt à nous accueillir, à recevoir l'homme et à lui donner la place centrale de la valeur, de la mesure, du jugement, du point de vue, de la perspective, etc.

11 La khôra est pour Derrida un lieu de résistance : elle résiste aussi bien à la maîtrise démiurgique qu'aux appropriations anthropomorphiques et symboliques auxquelles elle donne lieu sans pour autant s'y réduire. Elle est un espace d'exclusion et de sélection qui indique à la fois la nécessité de la limite et le caractère fictionnel de toute limite. Elle est le lieu où s'inscrit et se construit une structure supplémentaire d'exclusions (le bon/le mauvais, l'homme/l'animal, etc.) qui décide de l'appartenance et de la non appartenance à un " nous ", à une " communauté » ou une « collectivité ».

12 C'est au moment où la communauté se sent menacée ou risque de disparaître que les mécanismes d'exclusion se multiplient et les récits d'appartenance à un territoire, à une langue, deviennent plus insistants. Le contexte de crise ou de catastrophe évoqué dans le préambule du Timée permet, par-delà le caractère ontocosmologique du récit 
central du dialogue, de dégager une autre scène dans laquelle la fiction des origines se lie à la production et à la survivance du meilleur genre humain et à une géopolitique des lieux.

13 Le motif de la semence, de la khôra en tant que crible qui sépare les bonnes semences des mauvaises, s'annonce déjà d'une certaine manière dans le début du Timée. Ici, la semence est à la base d'un discours sur le genos athénien et la survivance des meilleurs citoyens. Selon le prêtre égyptien, c'est grâce au fait « [qu']un peu de leur semence s'est conservé " que la descendance des Grecs a survécu aux catastrophes. Nés de la meilleure semence, fils de ceux qui vainquirent les envahisseurs atlantes, les Athéniens de la Grèce contemporaine à Solon ne savent pas que « la race la meilleure et la plus belle parmi les hommes" est née dans leur pays, car ils n'ont pas l'écriture pour pouvoir témoigner des exploits de leurs ancêtres ${ }^{20}$. Pourtant, sans le savoir, les Athéniens sont descendants de la meilleure race humaine dont la semence a survécu miraculeusement aux catastrophes cycliques qui assiégèrent le territoire. La terre, le pays, la khôra, a conservé la semence dont les Athéniens descendent ; la terre porte en elle le germe de la renaissance ou de la résurrection. "L'affirmation nationale - écrit Derrida - n'est jamais le constat d'un fait, c'est l'affirmation d'une renaissance promise à partir d'une mort et de la disparition du corps du mort ${ }^{21}$. Il y a affirmation nationale là où il n'y a jamais de la nation. La nation, n'ayant pas de lieu propre à elle, ne peut que se produire d'elle-même dans une dimension qui est à la fois fictionnelle et absolument vraie. C'est un des grands paradoxes du Timée : dans une chaîne des fictions narratives où il n'est jamais question de vérité mais seulement de vraisemblance ou de mythe vraisemblable, le récit que Critias rapporte à Socrate sur les origines de l'Athènes et de la guerre contre l'Atlantide est « absolument vrai $»^{22}$. La généalogie est alors mobilisée pour accréditer la vérité d'un récit dont la propriété réside, selon Socrate, en ceci que ce n'est pas une "simple fiction» mais " un haut fait réellement et anciennement accompli par cette cité $\aleph^{23}$. La scène catastrophiste dans laquelle cette affirmation «nationale » - à la fois politique et naturelle - a lieu n'est pas arbitraire. L'affirmation nationale est un appel aux ressources humaines et naturelles du territoire pour faire face à la menace extérieure et surmonter la calamité. C'est parce que le corps propre de la nation n'existe pas "au présent", qu'il est déjà entamé par le processus de sa disparition, «que l'affirmation nationale prend son élan et sa force ; sa force [continue Derrida] tient à l'absence de nation $»^{24}$. C'est "quand la nation n'existe pas ou est menacée de ne pas exister que l'affirmation nationale se tend $»^{25}$. Elle est toujours "l'affirmation d'un futur antérieur ${ }^{26}$ qui n'a jamais eu lieu et qu'il faut restituer régulièrement aussi bien par le discours que par des pratiques ritualisées de nettoyage et de purifications censées reconstituer l'unité du territoire. La khôra, sorte de nettoyeuse universelle, figure d'une sélection ayant lieu dans un passé immémorial, est tout aussi bien la condition de possibilité d'un "nous " - qu'il se détermine comme nation, race, genre, communauté ou autre - que le lieu qui témoigne du caractère à la fois nécessaire et fictif de toute frontière et de toute sélection. 


\section{BIBLIOGRAPHIE}

Derrida Jacques, « La Pharmacie de Platon », in La Dissémination, Paris, Seuil, 1972.

Derrida Jacques, Positions, Paris, Minuit, 1972.

Derrida Jacques, « Comment ne pas parler », in Psyché, Paris, Galilée, 1987.

Derrida Jacques, « Khôra », in Poikilia. Études offertes à Jean-Pierre Vernant, Paris, Éd. de l'École des hautes études en sciences sociales, 1987.

Derrida Jacques, Khôra, Paris, Galilée, 1993.

Derrida Jacques, Eisenman Peter, Chora L Works, New York, Monacelli Press, 1997.

Derrida Jacques, Foi et savoir [1995], Paris, Seuil, 2000.

Derrida Jacques, Voyous, Paris, Galilée, 2003.

Heidegger Martin, Introduction à la métaphysique, G. Kahn (trad.), Paris, Gallimard, 1967.

Loraux Nicole, Les Enfants d'Athéna, Paris, Seuil, 1990.

Platon, « Timée », in CEuvres complètes, t. X, A. Rivaud (trad.), Paris, Les Belles Lettres, 1925.

Platon, « Ménexène ", in Cuvres Complètes, Luc Brisson (trad.), Paris, Flammarion, 2008.

Regazzoni Simone, Nel nome di Chōra. Da Derrida a Platone e al di là, Genova, Il Melangolo, 2008.

Robin Léon, Platon [PUF, 1935], Paris, Quadrige, 1997.

\section{NOTES}

1. Jacques Derrida, Khôra, Paris, Galilée, 1993, p. 95-96.

2. Platon, «Timée », 50d, in Euvres complètes, t. X, A. Rivaud (trad.), Paris, Les Belles Lettres, 1925.

3. Ibid., 48e.

4. Léon Robin, Platon [PUF, 1935], Paris, Quadrige, 1997, p. 170.

5. Martin Heidegger, Introduction à la métaphysique, G. Kahn (trad.), Paris, Gallimard, 1967, p. 76.

6. Jacques Derrida, Khôra, Paris, Galilée, 1993, p. 17.

7. Ibid., p. 18.

8. Il n'y a pas de vérité de la khôra ou sur la khôra. C'est pourquoi, dans Khôra, Derrida insiste sur la nécessité de laisser le nom de khôra « à l'abri de toute traduction » (p. 23), aussi bien que de supprimer l'article défini qui « présuppose l'existence d'une chose, l'étant khôra auquel, à travers un nom commun, il serait facile de se référer » (p. 29-30).

9. Jacques Derrida, La Dissémination, Paris, Seuil, 1972, p. 199.

10. La première version de Khôra parut en 1987, dans Poikilia. Études offertes à Jean-Pierre Vernant, Paris, Éd. de l'École des hautes études en sciences sociales.

11. Derrida aurait abordé la question de la khôra au cours d'un séminaire tenu à l'École normale supérieure en 1972 (voir Jacques Derrida, Positions, Paris, Minuit, 1972, p. 101) et au cours de plusieurs séances lors d'un séminaire non identifié en 1985. C'est à ce dernier séminaire dont la transcription de Derrida a été conservée à l'Institut mémoires de l'édition contemporaine (DRR 175 «Chôra/Nomos, topos, logos ») que nous allons nous référer tout à l'heure.

12. Derrida s'occupe de la khôra de manière approfondie dans «Comment ne pas Parler " (Psyché, 1987), Khôra (1993), Foi et savoir (1995) et Chora L Works (1997). Les références à la khôra sont 
pourtant nombreuses depuis la «Pharmacie de Platon» (La Dissémination, 1972) jusqu'à Voyous (2003). Pour un rapport exhaustif, voir Simone Regazzoni, Nel nome di Chōra. Da Derrida a Platone e al di là, Genova, Il Melangolo, 2008, p. 15.

13. Jacques Derrida, Psyché, Paris, Galilée, 1987, p. 502.

14. Platon, Timée, $18 \mathrm{~d}-\mathrm{e}$.

15. Ibid., 48a-b.

16. Ibid., $51 \mathrm{~b}$.

17. Institut mémoires de l'édition contemporaine, DRR 175. Voir aussi Nicole Loraux, Les Enfants d'Athéna, Paris, Seuil, 1990, p. 41-42.

18. Platon, « Ménexène », 238a, in Euvres Complètes, Luc Brisson (trad.), Paris, Flammarion, 2008.

19. Ibid., 237d.

20. Platon, Timée, 23b-c.

21. Institut mémoires de l'édition contemporaine, DRR 175.

22. Platon, Timée, $20 \mathrm{~d}$.

23. Platon, Timée, 26c.

24. Institut mémoires de l'édition contemporaine, DRR 175.

25. Ibid., DRR 175.

26. Ibid., DRR 175.

\section{RÉSUMÉS}

Dans cet article, nous proposons de suivre l'interprétation de Jacques Derrida de la khôra du Timée afin d'élaborer une problématique de l'espace qui prenne en compte les aspects idéologiques et politiques qui sont à l'œuvre dans la pensée de Platon. Contrairement aux interprétations métaphysiques qui considèrent la khôra comme une entité éminemment passive, Derrida souligne que, dans le Timée, la khôra fonctionne comme un mécanisme de sélection qui, hétérogène à l'ordre de l'intelligible, ne peut être approché que de manière paradoxale et anachronique.

\section{INDEX}

Mots-clés : khôra, lieu, espace, mère, paradoxe, sélection, nécessité, anachronisme, analogie, exclusion, fiction, nation 